\title{
MEROMORPHIC MAPS IN THE NEVANLINNA CLASS
}

\author{
R. E. MOLZON AND G. PATRIZIO
}

\begin{abstract}
A meromorphic mapping from a relatively compact domain $D$ in a complex manifold to complex projective space is shown to be in the Nevanlinna class provided the mapping omits a set of hyperplanes of positive capacity. As a consequence, such mappings have admissible limits almost everywhere on $\partial D$.
\end{abstract}

In this note we give a sufficient condition that a meromorphic mapping of a relatively compact domain in a complex manifold be in the Nevanlinna class. As a consequence we obtain new results on the existence of boundary values of meromorphic mappings. A well-known result of Stein [5] says that a holomorphic function in the Nevanlinna class for a smooth bounded domain in $\mathbf{C}^{n}$ has admissible limit almost everywhere on the boundary of the domain. In a recent paper Lempert [1] extended this result to meromorphic functions. The second author showed the existence of admissible limits almost everywhere for meromorphic mappings in the Nevanlinna class [4].

For holomorphic mappings $F: B^{n} \rightarrow \mathbf{C}^{n}$ of the ball, a sufficient condition for $F$ to be in the Nevanlinna class may be given in terms of capacity [3]. As we shall see below this result extends to meromorphic mappings of arbitrary relatively compact domains with smooth boundary in a complex manifold. One key point in this discussion is the fact that it is not in general possible to write a meromorphic mapping in the Nevanlinna class with given components as quotients of holomorphic functions in the Nevanlinna class in dimensions greater than one.

Let $M$ be a connected complex manifold of dimension $m>1$ and $\chi$ a smooth strictly positive form of bidegree $(m-1, m-1)$ on $M$. Suppose $D \subset M$ is open, relatively compact, and with smooth boundary. We suppose $\psi$ is a defining function for $D$ so

(i) $D=\{x \in M: \psi(x)<0\}$,

(ii) $\partial D=\{x \in M: \psi(x)=0\}$,

(iii) $\psi$ is smooth up to the boundary and $d \psi \neq 0$ on $\partial D$.

Let

$$
D(r)=\{x \in M: \psi(x)<r\} \quad \text { for } r<0 .
$$

If

$$
X=\left[X_{0}: \cdots: X_{n}\right] \in \mathbf{P C}, \quad A=\left[A_{0}: \cdots: A_{n}\right] \in \mathbf{P}^{n} \mathbf{C}^{*},
$$

let

$$
\|A: X\|=\left|A_{0} X_{0}+A_{1} X_{1}+\cdots+A_{n} X_{n}\right| \backslash|X||A| .
$$

For $A \in \mathbf{P}^{n} \mathbf{C}^{*}$, let $H^{A}$ denote the hyperplane defined by

$$
H^{A}=\left\{Z \in \mathbf{P}^{n} \mathbf{C}:\|Z: A\|=0\right\} .
$$

Received by the editors January 14, 1983.

1980 Mathematics Subject Classification. Primary 32A15, 32A30; Secondary 32H25, 32J25. 
Let $F: D \rightarrow \mathbf{P}^{n} \mathbf{C}$ be a meromorphic mapping. The value distribution functions associated with $F$ are given below.

$$
T(r)=\int_{D(r)}(r-\psi) F^{*} \Omega \wedge \chi \quad \text { for } r_{0} \leq r<0, r_{0} \text { fixed. }
$$

The $(1,1)$ from $\Omega$ is the Kähler form of the Fubini-Study metric on $\mathbf{P}^{n} \mathbf{C}$.

$$
\begin{gathered}
m(r, A)=\int_{\partial \mathcal{D}(r)} \log \frac{1}{\|F: A\|}\left(2 d^{c} \psi \wedge \chi\right) \\
=\int_{\partial \mathcal{D}(r)} F^{*}\left(\log \frac{1}{\|Z: A\|}\right) \cdot\left(2 d^{c} \psi \wedge \chi\right) \\
d^{c}=\frac{\sqrt{-1}}{2 \pi}(\bar{\partial}-\partial) .
\end{gathered}
$$

Given $A \in \mathbf{P}^{n} \mathbf{C}^{*}$, let $\nu_{F}^{A}$ denote the $A$-divisor of $F$ and $S_{A}$ the support of $\nu_{F}^{A}$. The integrated counting function is then defined by

$$
N(r, A)=\int_{D(r) \cap S_{A}}(r-\psi) \nu_{F}^{A} \chi, \quad r_{0} \leq r<0 .
$$

The deficit is given by

$$
D(r, A)=\int_{D(r) \backslash D\left(\overline{r_{0}}\right)} \log \frac{1}{\|F: A\|} d\left(2 d^{c} \psi \wedge \chi\right) .
$$

The First Main Theorem (FMT) then states [6 or 7]

$$
T(r)+D(r, A)=N(r, A)+m(r, A)-m\left(r_{0}, A\right) .
$$

The functions appearing in the FMT above depend on the choices of $\psi$ and $\chi$; however, the boundedness of $T(r)$ is independent of the choices. The following definition is therefore independent of the functions $\psi$ and $\chi$.

Definition. $F: D \rightarrow \mathbf{P}^{n} \mathbf{C}$, a meromorphic mapping, is said to be in the Nevanlinna class, $N(D)$, provided $T(r)$ is bounded.

We are interested in obtaining a sufficient condition that a meromorphic mapping be in $N(D)$ in terms of value distribution properties of $F$. We use the following Blaschke type condition.

DEFinition. $F: D \rightarrow \mathbf{P}^{n} \mathbf{C}$, a meromorphic mapping, satisfies the Blaschke condition for $A$ provided $N(r, A)$ is bounded as a function of $r$.

The value distribution property of $F$ we use involves the capacity of a set of hyperplanes. Making the identification of hyperplanes in $\mathbf{P}^{n} \mathbf{C}$ with points in $\mathbf{P}^{n} \mathbf{C}^{*}$ we define the capacity of a Borel measurable set $E$ as:

DEFinition. If $E \subset \mathbf{P}^{n} \mathbf{C}^{*}$ let

$$
V(E)=\inf _{\mu \in \mathcal{P}(E)} \sup _{Z \in \mathbf{P}^{n} \mathbf{C}} \int_{A \in E} \log \frac{1}{\|Z: A\|} d \mu(A), \quad C(E)=1 / V(E),
$$

where $P(E)$ denotes the set of probability measures supported on $E$. For properties of this capacity see [2].

We can now give a sufficient condition that a meromorphic mapping be in the Nevanlinna class. 
THEOREM. With $D \subset M, \psi$ and $\chi$ as above, let $F: D \rightarrow \mathbf{P}^{n} \mathbf{C}$ be a meromorphic mapping. Suppose $F$ satisfies the Blaschke condition for all $A \in E$ with $C(E)>0$. Then $F \in N(D)$.

Proof. Since $N(r, A)<\infty$ for all $A \in E$ and $C(E)>0$, there exists a set $E_{0} \subset E$ with $N(r, A) \leq M_{0}$ for all $A \in E_{0}$ and $C\left(E_{0}\right)>0$. Let $\mu_{0}$ be an equilibrium measure associated with $E_{0}$. Integrate the FMT with respect to $\mu_{0}$ to get

Since

$$
\begin{aligned}
T(r)= & \int_{E_{0}} N(r, A) d \mu_{0}(A)+\int_{E_{0}} m(r, A) d \mu_{0}(A) \\
& -\int_{E_{0}} m\left(r_{0}, A\right) d \mu_{0}(A)-\int_{E_{0}} D(r, A) d \mu_{0}(A) .
\end{aligned}
$$

$$
\int N(r, A) d \mu_{0}(A) \leq M_{0}
$$

we need only show that the other integrals are finite. We show that the last integral is finite; the others follow in a similar manner.

$$
\begin{aligned}
\int_{E_{0}} D(r, A) d \mu_{0}(A) & =\int_{E_{0}} \int_{D(r) \backslash D\left(\overline{r_{0}}\right)} F^{*}\left(\log \frac{1}{\|Z: A\|}\right) d\left(2 d^{c} \psi \wedge \chi\right) d \mu_{0}(A) \\
& =\int_{D(r) \backslash D\left(\overline{r_{0}}\right)}\left[F^{*} \int_{E_{0}} \log \frac{1}{\|Z: A\|} d \mu_{0}(A)\right] d\left(2 d^{c} \psi \wedge \chi\right) .
\end{aligned}
$$

Since $C\left(E_{0}\right)>0$, we have the estimate

Hence

$$
\int_{E_{0}} \log \frac{1}{\|Z: A\|} d \mu_{0}(A) \leq V\left(E_{0}\right)=1 / C\left(E_{0}\right)
$$

$$
\int_{E_{0}} D(r, A) d \mu_{0}(A) \leq \int_{D(r) \backslash \mathcal{D}\left(\overline{r_{0}}\right)} 1 / C\left(E_{0}\right) \cdot d\left(2 d^{c} \psi \wedge \chi\right) \leq 1 / C\left(E_{0}\right) \cdot k,
$$

where $k$ is independent of $r$ (but depends of course on the choices of $\psi$ and $\chi$ ).

In [4], the second author shows that meromorphic mappings in the Nevanlinna class have admissible limits almost everywhere $\left(2 m-1\right.$ Hausdorff measure $\left.\nvdash_{2 m-1}\right)$ on $\partial D$. We therefore have immediately the following result.

Corollary. Let $D \subset M$ be as above. Suppose $F: D \rightarrow \mathbf{P}^{n} \mathbf{C}$ is a meromorphic mapping such that $F$ satisfies the Blaschke condition for all $A \in E \subset \mathbf{P}^{n} \mathbf{C}^{*}$ with $C(E)>0$. Then $F$ has admissible limits almost everywhere $\mathfrak{H}_{2 m-1}$ on $\partial D$.

REMARKS. It is possible to localize the above results in the following sense. If $D \subset M$ is any domain with smooth boundary, choose $\rho \in D$. Then $F: D \rightarrow \mathbf{P}^{n} \mathbf{C}$ meromorphic is in the local Nevanlinna class at $\rho, N_{\text {loc }}(\rho, D)$, if there exists a coordinate neighborhood $U \subset M$ about $\rho$ such that $F \in N(D \cap U)$. One may then formulate local versions of the above results. See Stein [5] for a comparison.

\section{REFERENCES}

1. L. Lempert, Boundary behavior of meromorphic functions of several variables, Acta Math. 144 (1980), 1-25.

2. R. Molzon, B. Shiffman and N. Sibony, Average growth estimates for hyperplane sections of entire analytic sets, Math. Ann. 257 (1981), 43-59. 
3. R. Molzon, Holomorphic mappings in the Nevanlinna class, Math. Z. (to appear).

4. G. Patrizio, Boundary behavior of meromorphic maps, Math. Ann. 261 (1982), 111-132.

5. E. Stein, Boundary behavior of holomorphic functions of several complex variables, Princeton Univ. Press, Princeton, N.J., 1972.

6. W. Stoll, Deficit and Bezout estimates: Value distribution theory, Part B (Edited by R. Kujula and A. Vitter III), Pure and Appl. Math., Vol. 25, Marcel Dekker, New York, 1973.

7. __ The Ahlfors-Weyl theory of meromorphic maps on parabolic manifolds (Proc. Nordic Summer School, Joensuu, Finland, 1981), Lecture Notes in Math., Springer-Verlag, Berlin, Heidelberg, New York, 1982.

Department of Mathematics, University of KentuCky, Lexington, KenTUCKY 40506 (Current address of R. E. Molzon)

Department of Mathematics, University of Notre Dame, Notre Dame, INDiANA 46556

Current address (G. Patrizio): Max-Planck-Institut für Mathematik, Gottfried-Claren Strasse 26, 5300 Bonn 3, BRD (FRG) 